\title{
Le langage oral en production chez les enfants bilingues : quels liens avec l'exposition?
}

\author{
Cathy Cohen ${ }^{1,2,3,{ }^{*}}$, Audrey Mazur-Palandre ${ }^{1,2}$ \\ ${ }^{1}$ Laboratoire ICAR (UMR5191, CNRS, Université Lyon 2 et ENS de Lyon), 15 parvis René \\ Descartes BP 7000, 69342 Lyon Cedex 07 \\ ${ }^{2}$ Laboratoire d'Excellence ASLAN, (ANR-10-LABX-0081, de l'Université de Lyon dans le \\ cadre du programme « Investissements d'Avenir » (ANR-11-IDEX-0007) de l'État Français \\ géré par l'Agence Nationale de la Recherche), 14 avenue Berthelot, 69363 Lyon Cedex 7 \\ ${ }^{3}$ ESPE, Université Lyon 1, 5 rue Anselme, 69004 Lyon
}

\begin{abstract}
Résumé. L'objectif de cette étude est d'observer les effets de l'exposition aux langues sur différentes variables linguistiques en production orale d'enfants bilingues français/anglais sans pathologie, scolarisés dans une école internationale publique (France), et répartis en deux groupes d'âges (groupe 1: $N=10, M=6 ; 3$; groupe 2: $N=15, M=10 ; 2$ ). Des questionnaires parentaux ont permis d'obtenir des mesures quant à l'exposition courante et cumulative aux langues ainsi qu'à la fréquence à laquelle les enfants lisent dans les deux langues. À partir de tests de vocabulaire réceptif standardisés et de productions narratives (Frog, where are you?), des données linguistiques françaises et anglaises ont été obtenues. Les narrations ont été transcrites en respectant les conventions CHILDES dans le logiciel CLAN, permettant l'observation de diverses mesures linguistiques relatives au lexique, à la morphosyntaxe et au discours. Des corrélations ont été effectuées entre les mesures d'exposition et celles des performances linguistiques. Les résultats révèlent que le vocabulaire réceptif et certaines mesures de productivité narrative semblent être sensibles au degré d'exposition de chaque langue. De plus, nos conclusions renforcent l'idée du rôle essentiel de la lecture sur la performance linguistique, et ce d'autant plus pour les enfants les plus jeunes. Pour finir, cette étude souligne l'importance de travailler en étroite collaboration avec les équipes pédagogiques et les parents d'enfants bilingues afin de fournir des recommandations concrètes pour enrichir le langage oral.
\end{abstract}

\begin{abstract}
Oral language production in bilingual children: what links with exposure?

The study aims at examining oral language development in both languages of two groups of typically developing French/English bilingual children (group 1: $N=10, M=6 ; 3$; group $2: N=15, M=10 ; 2$ ) attending an international state school in France. We explore the effects of language exposure on several oral language performance variables. Parent questionnaires provide measures on current and cumulative exposure and frequency of book reading (shared reading for group 1; autonomous
\end{abstract}

\footnotetext{
* Corresponding author : cathy.cohen@ens-lyon.fr
} 
reading for group 2). French and English data are collected using standardised vocabulary tests and a story generation task (Frog, where are you?). Narratives are coded in the CHAT format of CHILDES to assess a range of narrative productivity measures relating to lexicon, morphosyntax and discourse. Correlations are computed between the language exposure and oral language performance measures. Overall, results provide further evidence that receptive vocabulary and certain narrative productivity measures are sensitive to exposure in each language. Most strikingly, frequency of book reading predicts language performance, particularly in younger children. The study highlights the importance of working closely with teachers and parents of bilingual children in order to provide concrete recommendations to enrich oral language.

\section{Introduction}

Il est largement admis que de nombreux facteurs contribuent à l'acquisition, au développement et au maintien du bilinguisme chez l'enfant (De Houwer, 2015 ; Pearson et Amaral, 2014). L'analyse des rôles joués par ces facteurs est compliquée par la nature dynamique et complexe du processus d'acquisition bilingue (Herdina et Jessner, 2002). Par conséquent, puisque les environnements linguistiques de l'enfant changent au fil du temps, les rôles joués par les différents facteurs évoluent, entraînant ainsi des modifications dans l'équilibre entre les deux langues, alors que l'enfant s'adapte à ses besoins communicatifs. Une des caractéristiques fondamentales du développement bilingue est donc la variabilité, à la fois en termes de durée d'exposition à chaque langue et de niveaux de compétence atteints dans chaque langue.

Cet article a pour objectif d'explorer, pour des individus bilingues, l'impact de l'exposition à une langue sur le langage oral de cette même langue, et ceci en nous appuyant sur des données provenant de la première année d'une recherche longitudinale actuellement en cours de recueil et prévue pour une durée de cinq ans, le projet INEXDEB (INput et EXpérience dans le DEveloppement Bilingue) (Cohen, 2015). Ce projet a pour principal objectif de réaliser une étude longitudinale sur le développement du langage oral des enfants bilingues, ce qui est encore bien trop rare (mais voir Jia et Aaronson, 2003 ; Scheides et Tuller, 2016). Les participants des analyses présentées dans ce présent papier sont des enfants bilingues (ou en devenir bilingues) français/anglais scolarisés dans une école publique en France, proposant plusieurs sections internationales, dont une section anglophone. Dans cette étude préliminaire, deux groupes sont observés : 10 enfants de $\mathrm{CP}$, première année de l'école élémentaire (groupe $1: N=10$, âge moyen $=6 ; 3$ ), et 15 enfants de $\mathrm{CM} 2$, dernière année de l'école élémentaire (groupe $2: \mathrm{N}=15$, âge moyen $=10 ; 2$ ).

\section{Cadre théorique}

\subsection{Facteurs d'exposition}

Les facteurs d'exposition influençant l'acquisition, le développement et le maintien du bilinguisme chez l'enfant peuvent être de deux ordres : ceux concernant les effets de la qualité et ceux concernant les effets de la quantité de l'exposition à chaque langue, appelé aussi input. En ce qui concerne la qualité de l'input, l'acquisition d'un bilinguisme équilibré nécessite une exposition à des modèles linguistiques de qualité, permettant à l'enfant d'avoir des contacts riches et variés dans ses deux langues (De Houwer, 2011). Cela comprend l'exposition à des locuteurs natifs et d'autres sources telles que la lecture (Paradis, 2011) (développé ci-dessous). Il est également admis que le niveau d'éducation 
de la mère a un impact fort sur le développement et l'enrichissement du lexique de l'enfant (Goldberg, Paradis et Crago, 2008).

Toutefois, c'est la quantité d'exposition à chaque langue qui est considérée comme un des facteurs critiques contribuant à l'acquisition bilingue (De Houwer, 2011 ; Pearson, 2007). De nombreuses études montrent que la quantité d'input à laquelle sont exposés les enfants d'âge préscolaire se révèle très prédicative de l'acquisition du vocabulaire dans chaque langue (Pearson, Fernández, Lewedeg et Oller, 1997) et qu'elle a également un impact sur la vitesse d'acquisition de certaines structures morphosyntaxiques complexes (Gathercole, 2007). Certaines études, qui ont cherché à évaluer le pourcentage d'exposition nécessaire à chaque langue permettant une utilisation aisée de chaque langue (Thordardottir, 2011), révèlent que les résultats aux évaluations de vocabulaire réceptif des bilingues ayant entre 40 et $60 \%$ d'exposition étaient proches de ceux des monolingues; néanmoins, sur les évaluations de vocabulaire productif, seuls les bilingues ayant plus de $60 \%$ d'exposition obtenaient des résultats équivalents aux monolingues. De plus, leurs heures d'éveil étant divisées entre deux langues, les enfants bilingues pourraient mettre plus de temps pour atteindre les normes monolingues. Toutefois, ils devraient finir par rattraper leur retard si l'exposition à chaque langue reste constante (Thordardottir, Rothenberg, Rivard et Naves, 2006). Si une exposition assez équilibrée aux deux langues est importante, produire (appelé output) dans les deux langues, l'est tout autant. En effet, Bohman et al. (Bohman, Bedore, Peña, Mendez-Perez, Gillam, 2010, p. 339) insistent sur le fait que 'using a language forces the learner to process the language in a way that only hearing it does not'. L'enfant doit alors sentir un réel besoin de parler ses deux langues à différents interlocuteurs, d'âges variés et dans des contextes sociaux divers (Pearson, 2008), y compris dans des domaines monolingues, et ce dans l'objectif d'apprendre à manipuler les différents registres des deux langues (Fishman, 2001).

De nombreuses études explorent les relations entre l'exposition courante à chaque langue (c'est-à-dire, l'exposition à un moment précis dans le présent) et divers indicateurs linguistiques. Toutefois une grande variation dans l'exposition à chaque langue peut exister chez un même individu bilingue au fil du temps. Par conséquent, il est aussi important d'évaluer l'exposition cumulative à chaque langue, c'est-à-dire de la naissance jusqu'au moment présent. Dans une étude sur des enfants bilingues français/anglais en CE1 (2 $2^{\text {ème }}$ année d'école élémentaire) en France, il a été démontré que l'exposition cumulative à chaque langue est très prédicative du niveau du vocabulaire réceptif et du langage oral en production dans chaque langue (Cohen, 2016). Des études complémentaires sont évidemment nécessaires pour déterminer le rôle de l'exposition cumulative sur l'acquisition d'un large éventail de structures linguistiques, objectif du projet INEXDEB (Cohen, 2015).

L'exposition aux langues peut aussi être évaluée en explorant l'exposition à des sources d'input riches (Paradis, 2011). L'exposition par la lecture est particulièrement pertinente, et débute bien avant la scolarisation, à la maison, avec les parents. De nombreuses études indiquent une relation très étroite entre la fréquence de lecture et le développement du langage oral dans son ensemble (Mol et Bus 2011; van Kleek et Stahl 2003), mais aussi entre la fréquence de lecture et le développement du vocabulaire (Mol, Bus et de Jong 2009 ; Sénéchal 2006). En effet, la lecture offre une exposition répétée à un vocabulaire riche et parfois rare, ce qui peut compléter celui rencontré dans le langage au quotidien durant, par exemple, les interactions parents/enfants (DeTemple, 2001 ; Weizman et Snow, 2001). Concernant les enfants bilingues, selon les pratiques langagières familiales, une langue peut être beaucoup moins présente à la maison ; ce qui est le cas, par exemple, des familles ayant une langue familiale principale différente de la langue majoritaire du pays. Pour ces enfants-là, il se peut que l'enrichissement lexical favorisé par la lecture à la maison ait lieu dans la langue familiale principalement alors que le vocabulaire dans la langue de scolarisation s'enrichit moins, ce qui peut avoir des répercussions sur la réussite scolaire (Oller et Eilers, 2002). 


\subsection{Compétences narratives orales}

De nombreuses études montrent des liens étroits entre le langage oral précoce et les acquisitions scolaires ultérieures telles que l'apprentissage de la lecture et le développement de la compréhension de l'écrit (entre autres, Bianco, 2015 ; Catts, Adolf et Weismer, 2006 ; Shanahan et Lonigan, 2013). Ainsi, le langage oral est ciblé de plus en plus dans les interventions auprès des lecteurs en difficulté (Lonigan, Schatschneider et Westberg, 2008). Avant de devenir lecteur, l'enfant acquiert des compétences en pré-lecture y compris des capacités à raconter une histoire à l'oral (NICHD, 2005). Les compétences narratives orales sont très prédicatives des compétences ultérieures en compréhension de l'écrit (GardnerNeblett et Iruka, 2015) et sont, par ailleurs, un prérequis pour l'apprentissage de l'écrit. Ainsi, elles sont indispensables pour le bon déroulement des apprentissages scolaires dans l'ensemble (Bliss, McCabe et Miranda, 1998). La production narrative semble donc être un excellent outil d'analyse de la productivité linguistique que ce soit au niveau des indicateurs linguistiques ou au niveau de la structure narrative, pour les enfants monolingues (Schneider, Hayward et Dubé, 2006) ou bilingues (Pearson, 2002). Des travaux se sont consacrés aux compétences narratives orales chez les enfants bilingues (entre autres, Altman, Armon-Lotem, Fichman et Walters, 2016 ; Gagarina, Klop, Tsimpli et Walters, 2016 ; Rodina, 2017 ; Simon-Cereijido et Gutiérrez-Clellen, 2009 ; Uccelli et Paéz, 2007). En effet, une narration orale, avec un support identique pour les deux langues, constitue des données linguistiques pertinentes, permettant l'étude du langage oral des enfants bilingues. Ce type de production permet ainsi de comparer de multiples compétences à l'intérieur d'une seule tâche, qui plus est écologique. La narration, par sa macrostructure essentiellement linéaire, son organisation temporelle (Berman et Slobin, 1994 ; Hickmann, 2003 ; Labov, 1993) et causale (Aksu-Koç et Küntay, 2001 ; Fayol, 2000 ; Jisa et Mazur, 2006) ainsi que sa structuration clairement identifiée (Labov, 1993), est un des types de textes acquis le plus précocement par les enfants. En effet, l'organisation linéaire du type de texte narratif répond à l'aspect antinomique de la production langagière. Tout message est verbalement linéaire alors que le modèle mental sous-jacent est, par définition, multidimensionnel. Il n'est en effet « qu'exceptionnellement linéaire » (Fayol, 1997, p. 146). Le texte narratif serait un des seuls types de textes à avoir un modèle mental pouvant être linéaire. C'est la raison pour laquelle les enfants maitriseraient plus précocement ce type de texte que tout autre (Fayol, 1984 ; Jisa, 2000, Ravid, 2000 ; Wigglesworth, 1991). Concernant la gestion textuelle de l'individu bilingue, la structure narrative et ainsi les éléments de macrostructure (cadre de l'histoire, objectifs, tentatives, résultats, réactions) semblent être transférés d'une langue à l'autre, appelant ainsi les mêmes processus cognitifs (Altman et al., 2016 ; Iluz-Cohen et Walters, 2012 ; Rodina, 2017 ; Uccelli et Paéz, 2007), comme l'indique également le Linguistic Interdependence Hypothesis (Cummins, 1978). En revanche, les différents éléments de productivité narrative (microstructure) dépendent davantage de la compétence linguistique dans chaque langue et sont souvent en étroite relation avec la quantité d'exposition à chaque langue (Pearson, 2002 ; Rodina, 2017 ; Rojas et Iglesias, 2013). Les études antérieures montrent que plusieurs mesures de microstructure linguistique sont pertinentes pour l'analyse des narrations de l'enfant : (1) la productivité lexicale mesurée par le nombre de mots différents et de verbes différents (entre autres, Pearson, 2002 ; Rodina, 2017 ; Rojas et Iglesias, 2013 ; Simon-Cereijido et Gutiérrez-Clellen, 2009); (2) les erreurs morphosyntaxiques (Bedore, Peña, Gillam et Ho, 2010 ; Pearson, 2002 ; Rodina, 2017); (3) la complexité syntaxique (Pearson, 2002; Rodina, 2017 ) et (4) la fluence mesurée par les marques de travail de formulation (nombre de répétitions et de reformulations), le débit du discours (Montanari, 2004 ; Rojas et Iglesias, 2013) et la durée des pauses vides (Candéa, 2000). 


\section{Questions de recherche}

L'objectif de cette présente étude est d'explorer les effets de l'exposition à une langue sur plusieurs mesures du langage oral en production. Les participants sont des enfants bilingues français/anglais scolarisés dans une école publique française. Un questionnaire rempli par les parents fournit des estimations sur le temps d'exposition, courante et cumulative, et de lecture pour chaque langue. Des données françaises et anglaises sont recueillies par le biais d'un test de vocabulaire standardisé et d'une tâche de narration, dans le but d'évaluer différentes mesures de productivité relatives au lexique, à la morphosyntaxe et au discours : le nombre de mots et de verbes différents, les erreurs morphosyntaxiques, le nombre de phrases complexes, les marques de travail de formulation, le débit du discours et la durée des pauses. La question de recherche est la suivante : quelle est la relation entre l'exposition à chaque langue et: 1- le vocabulaire réceptif et 2- divers aspects de la productivité narrative orale dans chaque langue?

Beaucoup d'études citées supra ont pour objectif principal d'explorer les relations entre différents éléments de microstructure au sein d'une même langue et entre les deux langues de l'enfant bilingue. Ainsi, alors que l'exposition est certes évoquée, elle n'est pas centrale, à la différence de notre étude, qui peut dès lors apporter donc une vision et des résultats complémentaires aux études précédemment citées. En effet, nous avons, tout d'abord, deux groupes d'enfants bilingues d'âges différents et scolarisés en école élémentaire, alors que la plupart des études citées s'intéressent aux enfants en école maternelle. Ensuite, le contexte de notre recherche, qui est explicité dans la partie 4.1, n'a, à notre connaissance, pas d'équivalent par rapport aux modèles connus de l'éducation bilingue (Baker, 2006). Par conséquent, la variété de profils des participants (cf. partie 4.2) fait que notre public est atypique et n'est donc pas, à notre connaissance, étudié dans la littérature. Enfin, dans la mesure où l'exposition a un impact fort sur le développement du langage oral, nous nous sommes efforcées de prendre en considération plusieurs mesures d'exposition précises : exposition courante, cumulative et fréquence de lecture.

Étant donnés les résultats des différentes recherches indiquées supra, nous postulons (H1), premièrement, que les mesures relatives à l'exposition seront prédicatives des résultats concernant le vocabulaire réceptif et des mesures concernant la productivité lexicale ainsi que les erreurs morphosyntaxiques dans les narrations (Pearson, 2002; Rodina, 2017; Rojas et Iglesias, 2013). Deuxièmement, nous pensons (H2) que l'exposition aura un impact moindre sur la construction de phrases complexes. En effet, les compétences mises en œuvre ici relèveraient davantage de compétences conceptuelles et cognitives générales que purement linguistiques (Pearson, 2002). Troisièmement, nous supposons (H3) que la durée d'exposition aura un impact sur les mesures de fluence. Si nous trouvons peu d'informations sur les relations entre l'exposition et la fluence chez les enfants bilingues (mais voir Montanari, 2004), les études sur l'acquisition des langues secondes par les adultes, montrent que les apprenants de niveaux linguistiques plus avancés ont généralement des productions orales ayant un débit plus rapide et contenant moins d'hésitations et de reformulations que les apprenants plus faibles (Yuan et Ellis, 2003).

\section{Méthodologie}

\subsection{Contexte}

Cette étude fait partie d'un projet de plus grande envergure, le projet INEXDEB (Cohen, 2015), qui répond à une demande du directeur et de l'équipe pédagogique d'une école élémentaire publique avec des sections internationales en France, qui manifestaient une 
volonté de mieux comprendre le développement linguistique des enfants bilingues, pour une meilleure prise en charge, notamment quand ces enfants présentent des difficultés. Cette école accueille des enfants du CP jusqu'à la Terminale et leur propose une forme forte d'éducation bilingue additive. Les trois quarts de la semaine scolaire sont consacrés aux programmes de l'Éducation Nationale française, le dernier quart étant consacré à l'enseignement en anglais de certains éléments de littéracie du National Curriculum britannique. Pour intégrer la section anglophone, un enfant doit avoir un niveau natif ou quasi-natif en anglais, qu'il est censé maintenir durant sa scolarité. L'école ne propose ni cours d'anglais langue étrangère, ni programmes enrichis en anglais pour aider ponctuellement des enfants en difficulté. Les enfants non-francophones qui arrivent de l'étranger suivent des cours de français langue seconde plusieurs heures par semaine pendant environ deux ans.

\subsection{Participants}

Pour ce présent papier, certains résultats aux questionnaires, les tests de vocabulaire réceptif et les productions narratives de dix enfants de $\mathrm{CP}$ et de 15 enfants de CM2 sont analysés. Le Tableau 1 résume les informations concernant ces deux groupes.

Tableau 1. Répartition des groupes selon l'âge et le genre

\begin{tabular}{|c|c|c|}
\hline & $\mathbf{C P}$ & CM2 \\
\hline \multicolumn{3}{|l|}{ Âge } \\
\hline Moyenne & $6 ; 3$ & $10 ; 2$ \\
\hline Intervalle & $5 ; 9-6 ; 8$ & $9 ; 10-10 ; 8$ \\
\hline Nombre total d'individus & 10 & 15 \\
\hline Nombre de filles & 7 & 9 \\
\hline Nombres de garçons & 3 & 6 \\
\hline
\end{tabular}

Les parents ont dans l'ensemble un haut niveau d'instruction (Tableau 2), informations collectées par le biais du questionnaire parental.

Tableau 2. Education des parents

\begin{tabular}{lccccc}
\hline & Niveau Bac + 2 & Niveau Bac + 3 & $\begin{array}{c}\text { Niveau Bac + 5 } \\
\text { et plus }\end{array}$ & Autre & Total \\
\hline Mère & 1 & 3 & 21 & - & 25 \\
\hline Père & 2 & 5 & 16 & 1 & 24 \\
\hline
\end{tabular}

Les situations linguistiques des familles des participants sont de trois types :

- $\quad$ un parent francophone natif et un parent anglophone natif $(N=9)$, dont la mère est anglophone dans 8 familles ;

- deux parents francophones natifs ayant vécu dans un pays anglophone avec leur enfant pendant au moins 3 ans $(N=6)$;

- deux parents anglophones natifs $(N=8)$, habitant en France depuis au moins 2 ans (excepté pour une famille qui venait d'arriver en France) ;

- d'autres configurations, impliquant une langue familiale autre que l'anglais ou le français, les parents ayant vécu dans un pays anglophone avec leur enfant avant d'arriver en France $(N=2)$.

Le contexte dans lequel les données ont été collectées (école bilingue en France scolarisant des enfants avec des parcours linguistiques très variés) et les profils familiaux des enfants sont une des spécificités de cette étude, et en ce sens elle peut être complémentaires aux travaux reportés jusqu'à présent dans la littérature. 


\subsection{Procédure et outils de recherche}

Les séances de travail avec les enfants ont été enregistrées avec l'accord écrit des parents. Chaque enfant a été rencontré individuellement cinq fois par an, lors de la pause déjeuner. Le Tableau 3 résume l'organisation des sessions de la collecte des données.

Tableau 3. Organisation des sessions

\begin{tabular}{cll}
\hline Session & \multicolumn{1}{c}{ Ordre anglais/français } & \multicolumn{1}{c}{ Ordre français/anglais } \\
\hline $\mathbf{1}$ & British Picture Vocabulary Scale & Échelle de vocabulaire en images Peabody \\
\hline $\mathbf{2}$ & Entretien sur les langues & Entretien sur les langues \\
\hline $\mathbf{3}$ & Échelle de vocabulaire en images Peabody & British Picture Vocabulary Scale \\
\hline $\mathbf{4}$ & Frog, where are you? français & Frog, where are you? anglais \\
\hline $\mathbf{5}$ & Frog, where are you? anglais & Frog, where are you? français \\
\hline
\end{tabular}

Chaque session a duré environ quinze minutes et il y avait au moins une semaine entre chaque session, excepté pour les sessions 4 et 5 , séparées de trois semaines minimum. L'ordre de passation des outils linguistiques dans les deux langues est contrôlé et contrebalancé. Tous les enfants ont fait toutes les activités dans les deux langues, sauf un en $\mathrm{CP}$ avec deux parents anglophones, en France depuis un mois au moment des passations, qui a fait les activités en anglais uniquement.

\subsubsection{Questionnaire parental}

Il a été demandé aux parents des enfants de remplir un questionnaire, dont une partie est consacrée à l'exposition des enfants au français et à l'anglais, et ce, en focalisant sur : 1l'exposition courante pendant la période scolaire et durant les vacances ; 2- 1'exposition cumulative de la naissance au moment de l'expérimentation et 3- la fréquence de lecture. Les questionnaires ont été informatisés et traités statistiquement avec SPSS.

L'exposition courante a été calculée en se basant sur les réponses des parents aux questions concernant l'estimation du nombre total d'heures par semaine durant lesquelles leur enfant était en contact avec chaque langue, dans un premier temps, durant les périodes scolaires (y compris les week-ends) et, dans un second temps, pendant les vacances scolaires. Le Tableau 4 fournit un exemple pour un élève de CP, participant à l'étude, du calcul de l'exposition quotidienne pendant la période scolaire. Le total d'heures en contact avec chaque langue est multiplié par 36, le nombre de semaines passées à l'école, (ligne 10 du Tableau 4).

Tableau 4. Exposition quotidienne pendant la période scolaire (Nombre d'heures en contact avec l'anglais, le français et d'autres langues éventuelles).

\begin{tabular}{|c|c|c|c|c|c|c|c|c|}
\hline & & $\begin{array}{l}\text { Anglais à } \\
\text { l'école }\end{array}$ & $\begin{array}{c}\text { Anglais en } \\
\text { dehors de } \\
\text { l'école }\end{array}$ & $\begin{array}{c}\text { Français à } \\
\text { l'école }\end{array}$ & $\begin{array}{c}\text { Français en } \\
\text { dehors de } \\
\text { l'école }\end{array}$ & $\begin{array}{c}\text { Autres } \\
\text { langues à } \\
\text { l'école }\end{array}$ & $\begin{array}{c}\text { Autres langues } \\
\text { en dehors de } \\
\text { l'école }\end{array}$ & $\begin{array}{c}\text { TOTAL } \\
\text { heures }\end{array}$ \\
\hline 1 & Lundi & 1.5 & 2.5 & 6 & 2 & - & - & 12 \\
\hline 2 & Mardi & & 2.5 & 7.5 & 2 & - & - & 12 \\
\hline 3 & Mercredi & 3 & 5.5 & 1 & 2.5 & - & - & 12 \\
\hline 4 & Jeudi & 1.5 & 2.5 & 6 & 2 & - & - & 12 \\
\hline 5 & Vendredi & & 2.5 & 7.5 & 2 & - & - & 12 \\
\hline 6 & Samedi & & 3 & & 11 & - & - & 13 \\
\hline 7 & Dimanche & & 3 & & 11 & - & - & 13 \\
\hline 8 & TOTAL & 6 & 21.5 & 28 & 30.5 & - & - & 86 \\
\hline 9 & & \multicolumn{2}{|c|}{ Total anglais : $27.5 \mathrm{~h}$} & \multicolumn{2}{|c|}{ Total français : $58.5 \mathrm{~h}$} & \multicolumn{2}{|r|}{-} & \\
\hline 10 & & \multicolumn{2}{|c|}{ x 36 semaines $=990 \mathrm{~h}$} & \multicolumn{2}{|c|}{$\mathrm{x} 36$ semaines $=2106 \mathrm{~h}$} & \multicolumn{2}{|r|}{-} & \\
\hline
\end{tabular}

Le Tableau 5 fournit un exemple pour le même élève en CP de l'exposition quotidienne pendant les vacances scolaires. Le calcul est réalisé de la même manière que pour 
l'exposition quotidienne pendant la période scolaire (Tableau 4). Le total pour chaque langue est ensuite multiplié par 16, le nombre de semaines de vacances scolaires annuelles.

Tableau 5. Exposition quotidienne pendant les vacances scolaires (Nombre d'heures en contact avec l'anglais, le français et d'autres langues éventuelles).

\begin{tabular}{|l|c|c|c|c|}
\hline & Anglais & Français & Autres langues & TOTAL \\
\hline Lundi & 4 & 9 & - & 13 \\
\hline Mardi & 4 & 9 & - & 13 \\
\hline Mercredi & 4 & 9 & - & 13 \\
\hline Jeudi & 4 & 9 & - & 13 \\
\hline Vendredi & 4 & 9 & - & 13 \\
\hline Samedi & 4 & 9 & - & 13 \\
\hline Dimanche & 4 & 9 & - & 91 \\
\hline TOTAL & $28 \mathrm{~h}$ & $63 \mathrm{~h}$ & & 13 \\
\hline & $\mathrm{x} 16$ semaines $=448 \mathrm{~h}$ & $\mathrm{x} 16$ semaines $=1008$ & & \\
\hline
\end{tabular}

Le Tableau 6 montre le calcul final pour l'exposition courante à chaque langue pour l'élève en CP. Le total d'heures en contact avec chaque langue sur l'année est transformé en pourcentage pour faciliter les comparaisons entre les participants.

Tableau 6. Calcul de l'exposition courante totale

\begin{tabular}{lcc}
\hline & Anglais & Français \\
\hline Total heures période scolaire & 990 & 2106 \\
\hline Total heures période vacances & 448 & 1008 \\
\hline Total heures période scolaire + vacances & 1438 & 3114 \\
\hline TOTAL \% EXPOSITION COURANTE & $31.6 \%$ & $68.4 \%$ \\
\hline
\end{tabular}

L'exposition cumulative à chaque langue a été calculée à partir de différentes informations fournies par les parents sur les langues utilisées dans divers contextes (avec chaque membre de la famille proche, avec une nounou, à la crèche, à l'école), et ce pour chaque année, de la naissance au moment de l'expérimentation. Pour chaque année, une estimation de l'exposition à chaque langue a été calculée en pourcentage. Par exemple, si nous avons estimé que l'enfant a eu 60\% d'exposition à l'anglais et $40 \%$ d'exposition au français dans une année donnée, les totaux pour cette année seraient 0.6 et 0.4 respectivement (méthode de calcul adapté de Unsworth, 2013). Faisant la somme des résultats année par année pour un enfant de sept ans, si l'exposition à chaque langue reste constante, on arrive à une exposition cumulative à l'anglais de 4.2 années et au français, de 2.8 années. Les calculs se basent sur le nombre d'années en contact avec chaque langue et prennent donc en considération les âges différents des participants. Ces estimations reposent sur les représentations rétrospectives des parents, qui semblent fournir des informations fiables et correspondant relativement bien à la réalité (Paradis, Emmerzael et Duncan, 2010).

Pour calculer la fréquence de lecture dans chaque langue, nous nous sommes basées sur les réponses des parents aux questions concernant la lecture (Tableau 7): à quelle fréquence les parents lisent un livre (ou tout autre support de lecture) à leur enfant et à quelle fréquence l'enfant lit en autonomie (nombre de fois par an, mois et semaine). Pour le groupe de CP, cela concerne donc les temps de lecture partagée avec les parents et pour le groupe de CM2 la lecture en autonomie.

Tableau 7. Estimation de la fréquence de lecture dans chaque langue

\begin{tabular}{|l|c|c|c|c|c|c|}
\hline & Jamais & $\begin{array}{c}\text { Quelques } \\
\text { fois / an }\end{array}$ & $\begin{array}{c}1 \text { à 2 fois / } \\
\text { mois }\end{array}$ & $\begin{array}{c}1 \text { à 2 fois / } \\
\text { semaine }\end{array}$ & $\begin{array}{c}4 \text { à } 5 \text { fois / } \\
\text { semaine }\end{array}$ & $\begin{array}{c}\text { Quasiment tous } \\
\text { les jours }\end{array}$ \\
\hline Lecture autonome anglais & & & & & & \\
\hline Lecture autonome français & & & & & & \\
\hline Lecture partagée anglais & & & & & & \\
\hline Lecture partagée français & & & & & & \\
\hline
\end{tabular}


Les réponses cochées des parents ont ensuite été converties en estimations chiffrées de la fréquence de la lecture par an, en nombre de fois par an, pour faciliter les analyses statistiques (quelques fois par an $=8$ fois par an; 1 à 2 fois par mois $=20$ fois par an ; 1 à 2 fois par semaine $=80$ fois par an ; 4 à 5 fois $=230$ fois par an ; quasiment tous les jours $=$ 350 fois par an).

\subsubsection{Vocabulaire réceptif}

Les enfants ont passé un test de vocabulaire standardisé : le British Picture Vocabulary Scale (Dunn, Dunn, Whetton et Burley, 1987) et l'Échelle de Vocabulaire en Images Peabody (Dunn, Thérault-Whalen et Dunn, 1993). Les consignes des manuels ont été suivies; le chercheur lisait un mot et l'enfant indiquait l'image correspondante parmi les quatre présentées sur le support. Les scores bruts ont été normalisés selon l'âge.

\subsection{3 "Frog, where are you?»}

Les enfants ont également produit une narration orale en anglais et en français, élicitée à partir d'un album sans texte Frog, where are you ? (Mayer, 1969). Ce support comprend 24 images et retrace l'histoire d'un petit garçon qui perd sa grenouille puis part à sa recherche. Les mêmes consignes ont été prononcées pour chaque langue: Voilà une histoire d'un garçon, d'une grenouille et d'un chien. Tu vas d'abord regarder les images, et puis quand tu es prêt(e), tu vas me raconter l'histoire que tu vois sur les images. Les enfants étaient libres de tourner les pages quand ils le souhaitaient. Les données ont été enregistrées puis transcrites et codées dans le logiciel CLAN, selon les conventions CHILDES (MacWhinney, 2000). Étant données nos questions de recherche et nos hypothèses, nous nous sommes concentrées sur les variables linguistiques suivantes :

- la diversité lexicale (nombre de mots différents par rapport au nombre d'unités Terminales; nombre de verbes différents par rapport au nombre d'unités Terminales) ;

- la précision morphosyntaxique (la proportion d'erreurs morphosyntaxiques telles que des erreurs sur les déterminants, les pronoms, les accords ou encore la conjugaison, par rapport au nombre total de mots);

- la complexité syntaxique (la proportion de phrases complexes - phrase matrice principale et sa/ses subordonnée(s) - par rapport au nombre total de phrases) ;

- la fluence (la proportion de marques de travail de formulation, à savoir les répétitions et les reformulations par rapport au nombre total de mots; le nombre de mots par minute et la proportion de pauses vides (le seuil est de $250 \mathrm{~ms}$ ) par rapport à la durée totale).

Des tests de corrélation non paramétriques (Spearman Rho) ont été réalisés, entre les indicateurs d'exposition et les indicateurs linguistiques.

\section{Résultats}

Les statistiques descriptives pour les mesures relatives à l'exposition et les mesures linguistiques sont présentées en Annexe 1. Dans cette section, sont présentés les résultats concernant des corrélations non paramétriques entre l'exposition courante, l'exposition cumulative et la fréquence de lecture avec différents variables linguistiques. Les résultats significatifs sont marqués d'une étoile $*(p<.05)$ et ceux très significatifs de deux étoiles $* *$ $(p<.01)$. Dans un premier temps, des résultats concernant le vocabulaire réceptif sont présentés. S'ensuivent des résultats concernant les indicateurs linguistiques des narrations (diversité lexicale, précision morphosyntaxique, complexité syntaxique et fluence). 


\subsection{Vocabulaire réceptif}

Un test de corrélation a été réalisé entre le degré d'exposition courante, cumulative et le temps de lecture partagée et les résultats du test de vocabulaire réceptif (Tableau 8).

Tableau 8. Corrélations entre exposition / lecture et vocabulaire réceptif

\begin{tabular}{lcccc}
\hline & \multicolumn{2}{c}{ BPVS (anglais) } & \multicolumn{2}{c}{ EVIP (français) } \\
\hline & CP & CM2 & CP & CM2 \\
\hline Exposition courante & -.1 & $.51^{*}$ & $.65^{*}$ & .4 \\
Exposition cumulative & -.07 & .3 & $.76^{* *}$ & $.59^{*}$ \\
Lecture & $.64 *$ & .08 & $.64 *$ & -.14 \\
\hline
\end{tabular}

Les résultats révèlent que les relations entre l'exposition courante et cumulative et les résultats aux tests de vocabulaire réceptif sont plus fortes pour le français que pour l'anglais : il existe une relation entre exposition courante et cumulative et l'EVIP pour les $\mathrm{CP}$ et une relation entre exposition cumulative et l'EVIP pour les CM2. Pour l'anglais, les analyses révèlent une relation seulement entre l'exposition courante et le BPVS pour les CM2. Concernant la lecture, les résultats montrent une relation avec les tests de vocabulaire réceptif en français et en anglais pour les $\mathrm{CP}$, mais pas pour les $\mathrm{CM} 2$. Ces résultats vont dans le sens de notre première hypothèse $(\mathrm{H} 1)$ qui prévoyait une relation entre les mesures relatives à l'exposition et les mesures de vocabulaire réceptif, et ceci surtout pour les résultats en français et davantage pour les $\mathrm{CP}$.

\subsection{Les indicateurs linguistiques, "Frog, where are you ? »}

\subsubsection{Diversité lexicale}

Le Tableau 9 présente les résultats des tests de corrélation effectués entre l'exposition courante, cumulative et la lecture et le nombre de mots différents (NMD) et le nombre de verbes différents (NVD) dans les productions narratives.

Tableau 9. Corrélations entre exposition / lecture et nombre de mots et de verbes différents

\begin{tabular}{|c|c|c|c|c|c|c|c|c|}
\hline & \multicolumn{4}{|c|}{ Anglais } & \multicolumn{4}{|c|}{ Français } \\
\hline & \multicolumn{2}{|c|}{ NMD } & \multicolumn{2}{|c|}{ NVD } & \multicolumn{2}{|c|}{ NMD } & \multicolumn{2}{|c|}{ NVD } \\
\hline & $\mathrm{CP}$ & CM2 & $\mathrm{CP}$ & CM2 & $\mathrm{CP}$ & CM2 & $\mathrm{CP}$ & CM2 \\
\hline $\begin{array}{l}\text { Exposition } \\
\text { courante }\end{array}$ & -.32 & -.32 & -.57 & .25 & $.58 *$ & $.68 * *$ & $.68^{*}$ & $.59 * *$ \\
\hline $\begin{array}{l}\text { Exposition } \\
\text { cumulative }\end{array}$ & -.38 & -.29 & -.28 & .12 & $.72 *$ & $.72 * *$ & $.77 * *$ & $.55^{*}$ \\
\hline Lecture & $.64 *$ & -.33 & .14 & .26 & $.6 *$ & $.47 *$ & $.6^{*}$ & .27 \\
\hline
\end{tabular}

Les résultats ne montrent pas, en anglais, de lien entre l'exposition courante et cumulative et le nombre de mots et de verbes différents (CP et CM2). En revanche, l'exposition courante et cumulative, en français, semblent être de bons indicateurs du nombre de mots et de verbes différents pour les enfants des deux groupes d'âges. Pour la lecture, les résultats sont significatifs pour le nombre de mots différents pour les $\mathrm{CP}$ en anglais et en français. Le résultat est également significatif pour les $\mathrm{CP}$ pour le nombre de verbes différents en français. Pour les CM2, les résultats sont significatifs pour le nombre de mots différents seulement en français. Ces résultats corroborent $\mathrm{H} 1$, tout particulièrement pour le français.

\subsubsection{Précision morphosyntaxique}

Le Tableau 10 présente les résultats des tests de corrélation effectués entre l'exposition courante, cumulative et la lecture et la proportion d'erreurs morphosyntaxiques. 
Tableau 10. Corrélations entre exposition / lecture et erreurs morphosyntaxiques

\begin{tabular}{lcccc}
\hline & \multicolumn{2}{c}{ Erreurs anglais } & \multicolumn{2}{c}{ Erreurs français } \\
\hline & CP & CM2 & CP & CM2 \\
\hline Exposition courante & .52 & .16 & $-.68^{*}$ & $-.46^{*}$ \\
Exposition cumulative & -.01 & .01 & $-.7^{*}$ & $-.63^{* *}$ \\
Lecture & -.02 & -.13 & $-.76^{* *}$ & -.15 \\
\hline
\end{tabular}

Les analyses ne révèlent aucune corrélation significative pour l'anglais. En revanche, pour le français, l'exposition courante et cumulative sont en corrélation négative avec le nombre d'erreurs morphosyntaxiques pour le CP et les CM2 : plus l'exposition est élevée, moins les enfants font d'erreurs morphosyntaxiques. La lecture partagée en français a un impact significatif sur la proportion d'erreurs morphosyntaxiques pour les CP : au plus les enfants lisent, au moins ils font d'erreurs morphosyntaxiques. Ces résultats confirment H1 seulement pour le français.

\subsubsection{Complexité syntaxique}

Le Tableau 11 présente les résultats des tests de corrélation entre l'exposition courante, cumulative, la lecture et la proportion de phrases complexes : aucun n'est significatif.

Tableau 11. Corrélations entre exposition / lecture et phrases complexes

\begin{tabular}{lcccc}
\hline & \multicolumn{2}{c}{ Phrases complexes anglais } & \multicolumn{2}{c}{ Phrases complexes français } \\
\hline & CP & CM2 & CP & CM2 \\
\hline Exposition courante & -.03 & .24 & .39 & .06 \\
Exposition cumulative & -.16 & .24 & .57 & .02 \\
Lecture & -.18 & -.18 & .51 & -.05 \\
\hline
\end{tabular}

L'exposition courante, cumulative et la lecture ne sont pas liées à la proportion de phrases complexes dans les narrations, allant ainsi dans le sens de $\mathrm{H} 2$.

\subsubsection{Fluence}

Le Tableau 12 présente les résultats des tests de corrélation entre l'exposition courante, cumulative, la lecture et (1) la proportion des marques de travail de formulation (MDTF) par rapport au nombre total de mots, (2) le nombre de mots par minute et (3) la durée des pauses vides par rapport à la durée totale de la narration, dans les productions narratives.

Tableau 12. Corrélations entre exposition / lecture et fluence

\begin{tabular}{|c|c|c|c|c|c|c|c|c|c|c|c|c|}
\hline & \multicolumn{6}{|c|}{ Anglais } & \multicolumn{6}{|c|}{ Français } \\
\hline & \multicolumn{2}{|c|}{ MDTF } & \multicolumn{2}{|c|}{$\begin{array}{l}\text { Mots par } \\
\text { minute }\end{array}$} & \multicolumn{2}{|c|}{ Pauses } & \multicolumn{2}{|c|}{ MDTF } & \multicolumn{2}{|c|}{$\begin{array}{c}\text { Mots par } \\
\text { minute }\end{array}$} & \multicolumn{2}{|c|}{ Pauses } \\
\hline & $\mathrm{CP}$ & CM2 & $\mathrm{CP}$ & CM2 & $\mathrm{CP}$ & CM2 & $\mathrm{CP}$ & CM2 & $\mathrm{CP}$ & CM2 & $\mathrm{CP}$ & CM2 \\
\hline $\begin{array}{l}\text { Exposition } \\
\text { courante }\end{array}$ & .17 & -.23 & .45 & .27 & -.3 & -.19 & -.48 & $-.59 *$ & -.13 & .39 & .43 & -.15 \\
\hline $\begin{array}{l}\text { Exposition } \\
\text { cumulative }\end{array}$ & .03 & -.15 & $.57 *$ & .37 & -.41 & -.16 & $-.58^{*}$ & $-.59 * *$ & .03 & .39 & .25 & -.05 \\
\hline Lecture & -.23 & -.23 & -.06 & -.25 & .25 & .16 & $-.64 *$ & .05 & .24 & .36 & -.01 & -.4 \\
\hline
\end{tabular}

Concernant l'anglais, les analyses révèlent un seul résultat significatif : il existe une relation entre le débit du discours (mots par minute) et l'exposition cumulative en anglais, seulement pour les $\mathrm{CP}$. Concernant le français, pour les $\mathrm{CP}$, les analyses montrent une corrélation négative entre le nombre de MDTF et l'exposition cumulative et la lecture partagée : plus les enfants ont été exposés au français depuis la naissance et plus les parents leur lisent des histoires, moins ils font des MDTF. Pour les CM2, l'exposition courante et cumulative sont en relation négative, seulement, avec la proportion de MDTF : plus 
l'exposition est élevée, moins il y a des MDTF. En revanche, la quantité d'exposition n'a pas d'effet sur le nombre de mots par minute et la durée des pauses (CP et CM2). Ces résultats vont à l'encontre de H3 qui prévoyait une relation entre l'exposition et la fluence.

\section{Discussion}

Discussion des résultats : Quelle est la relation entre exposition et vocabulaire réceptif et productivité narrative?

Une information intéressante révélée par les analyses est le fait que les corrélations entre les mesures relatives à l'exposition (exposition courante et cumulative ; lecture) et les mesures du langage oral, pour les enfants de $\mathrm{CP}$ et de $\mathrm{CM} 2$, sont généralement plus importantes pour le français que pour l'anglais. Comme dit précédemment, un niveau natif ou quasinatif est exigé pour intégrer la section anglophone de l'établissement scolaire. Par conséquent, au moment du recueil des données, les niveaux en anglais, surtout des enfants de CP commençant leur scolarité, étaient plus élevés et homogènes qu'en français. En effet, tous les participants avaient eu suffisamment d'exposition courante et cumulative en anglais pour atteindre des niveaux de performance élevés, avec beaucoup moins de variabilité qu'en français. Toutefois, il est intéressant de noter des corrélations significatives en anglais pour les enfants de $\mathrm{CP}$ entre la fréquence de la lecture partagée et le vocabulaire réceptif (BPVS) et le nombre de mots différents dans les narrations. Ces résultats confirment les travaux antérieurs (Mol et al., 2009; Sénéchal, 2006) concluant que la lecture contribuerait à l'enrichissement du lexique, avec notamment une exposition à des mots parfois rares. L'attrition de l'anglais étant possible au fil des années, notamment pour les enfants ayant deux parents francophones, avec le français comme langue familiale principale, il est possible que les relations entre les mesures relatives à l'exposition et les mesures du langage oral soient plus fortes au fur et à mesure que l'étude progresse, à savoir au fur et à mesure que l'enfant évolue et que ses situations communicationnelles changent.

Les résultats sur le français révèlent une plus grande variabilité chez les participants par rapport à l'exposition au français (Annexe 1). Les analyses corrélationnelles révèlent ainsi des effets importants de l'exposition courante et cumulative ainsi que de la fréquence de lecture partagée sur le vocabulaire réceptif (EVIP) pour les enfants de $\mathrm{CP}$, alors que l'exposition cumulative est le seul indicateur significatif pour les CM2. Certains éléments de la productivité narrative, et plus spécifiquement ceux liés à la compétence linguistique (lexique et morphosyntaxe) semblent être très sensibles à la quantité d'exposition courante et cumulative pour les deux groupes de participants ; ces résultats confirment des travaux antérieurs (Pearson, 2002 ; Rodina, 2017 ; Rojas et Iglesias, 2013). Par ailleurs, tout comme pour l'anglais, la fréquence de la lecture partagée pour les enfants de CP semble avoir un impact fort sur les compétences linguistiques. L'impact de la lecture en autonomie parait moins important pour les élèves de CM2.

De plus, comme nous l'avions postulé $(\mathrm{H} 3)$, les différentes mesures relatives à l'exposition sont de bons indicateurs de la fréquence des marques de travail de formulation - exposition cumulative et lecture partagée pour les élèves de CP ; exposition courante et cumulative pour les élèves de CM2. La présence des MDTF peut alors être le reflet de certaines fragilités du système linguistique, entravant la construction du message oral. Par conséquent, l'enfant serait obligé de répéter certains éléments pour planifier son message et de reformuler lorsqu'il lui manque des éléments lexicaux ou morphosyntaxiques pour exprimer avec précision ce qu'il souhaite dire. Il serait intéressant dans une étude ultérieure d'examiner de plus près la quantité d'output produit par les enfants dans leur vie quotidienne afin de mieux comprendre si ceux qui ont l'occasion de produire davantage dans la langue cible ont un discours contenant moins de MDTF. Deux mesures de fluence, le nombre de mots par minute et la durée des pauses vides, semblent cependant moins sensibles à l'exposition que les MDTF : un enfant qui a une exposition importante au 
français n'a pas forcément un débit rapide et sans pauses ; de la même manière, un enfant ayant une exposition plus courte n'a pas forcément un débit plus lent ponctué de longues pauses. Il semblerait donc que certains enfants ont simplement un débit rapide, alors que d'autres prennent plus de temps pour composer leur discours, et ceci quel que soit le niveau de compétence et quelle que soit la durée d'exposition à la langue en question.

Enfin, les analyses révèlent que les corrélations entre les mesures relatives à l'exposition et les mesures du langage oral en français sont, en général, plus fortes pour les enfants de $\mathrm{CP}$ que pour ceux de CM2. Ces résultats laissent à penser que l'exposition cumulative au français, d'une durée beaucoup plus longue que celle des enfants de CP, a permis aux enfants de CM2 d'accumuler une masse critique de données linguistiques (Gathercole, 2007), ce qui pourra être vérifié lors des futures analyses longitudinales du projet INEXDEB (Cohen, 2015).

Pour finir, notons que les résultats ne montrent pas de relation entre l'exposition et la construction de phrases complexes, comme nous l'avions postulé $(\mathrm{H} 2)$.

\section{Discussion des résultats en lien avec le système pédagogique dans lequel évoluent les enfants.}

Deux observations finales peuvent être faites. Premièrement, certains résultats peuvent laisser penser que l'acquisition du français est parfois plus lente pour certains enfants et nous pouvons penser que ces enfants en question sont ceux ayant deux parents anglophones, ce qui peut entraîner des difficultés dans la partie française du programme scolaire (le français étant la langue de scolarisation principale). En effet, selon les estimations des parents fournies dans le questionnaire parental, ces enfants ont très souvent moins de $10 \%$ de contact avec le français en dehors de l'école, l'anglais étant la seule langue familiale, mais aussi la langue quasi exclusive pour la lecture, la télévision et la vie sociale. Ce contexte linguistique implique alors que certains enfants n'ont que peu ou pas de contact avec le français durant les week-ends et les vacances scolaires. Par ailleurs, une fois scolarisés dans cet établissement, ces enfants peuvent choisir des groupes d'amis anglophones, réduisant ainsi considérablement l'exposition au français pendant le temps scolaire. Ceci s'additionne à un enseignement français plus frontal (avec principalement des interactions dites verticales et peu d'interactions horizontales) et réduisant donc fortement les occasions de produire beaucoup d'output en classe (Osborn, 2009), alors que les études actuelles montrent l'importance de la production en langue cible pour le développement du langage oral (Bohman et al., 2010). C'est dans cette mesure que l'observation de ces enfants dans une perspective longitudinale pourra apporter des éclairages quant au développement de la langue non dominante (ici le français), qui, en raison d'une exposition moins importante, a été clairement moins développée que la langue dominante (l'anglais). Si l'enfant montre de meilleures compétences dans le français, il est ainsi intéressant de comprendre ce qui permet un tel développement. En revanche, si l'enfant ne montre pas une amélioration significative, il est important de proposer des pistes d'action aux parents et à l'établissement scolaire afin d'aider ces enfants en difficulté.

Deuxièmement, les résultats de cette étude démontre qu'une exposition insuffisante peut rendre vulnérable le développement des compétences lexicales et morphosyntaxiques, alors qu'une bonne narration orale nécessite un lexique riche et varié avec une morphosyntaxe correcte. Les compétences narratives orales sont essentielles pour le développement de la compréhension de l'écrit et pour l'apprentissage de la production écrite (Gardner-Neblett et Iruka, 2015). Il est donc important d'intervenir auprès des enfants qui sont plus en difficulté dans une de leurs langues de scolarisation pour enrichir les compétences lexicales et morphosyntaxiques afin d'assurer la réussite scolaire. Compte tenu de nos résultats, révélant de nombreuses corrélations significatives entre la lecture partagée parent-enfant et les mesures lexicales et morphosyntaxiques, notamment pour les enfants de CP, il semblerait qu'une piste potentiellement intéressante à explorer serait la mise en place 
d'ateliers de lecture partagée à l'école. Ces ateliers intégreraient des caractéristiques de la lecture partagée parent-enfant : une lecture interactive ponctuée d'explicitations et de questions ouvertes, favorisant ainsi la production d'output de la part de l'enfant, sur l'histoire lue, que ce soit sur le vocabulaire ou sur le contenu (Aram, Fine et Ziv, 2013). La rétroaction donnée par le parent, est, de plus, très importante. Toutes ces caractéristiques favorisent l'enrichissement et la complexification du langage oral (Sénéchal, Pagan, Lever et Ouellette, 2008) et aide l'enfant à acquérir des compétences essentielles pour la compréhension de l'écrit (Sénéchal et Kearnan, 2007). Des travaux récents (PollardDurodola, Gonzalez, Simmons et Simmons, 2015) révèlent que, si les enseignants sont formés à la lecture partagée interactive et que ces techniques sont mises en œuvre en classe, un effet positif sur le lexique et les compétences narratives des élèves est alors constaté.

Si les enseignants peuvent aider les enfants bilingues à davantage développer la langue la plus fragile, le rôle des parents est également important. Certains enfants de l'étude ont une langue familiale principale, qui laisse peu de place à la deuxième langue. Les parents peuvent penser ne pas avoir une maîtrise suffisante de l'autre langue de scolarisation pour mettre en place une activité de lecture avec leur enfant ; alors que pour d'autres, il ne leur paraît pas naturel de lire dans une langue qui n'est pas leur langue maternelle. Par conséquent, la lecture à la maison se fait principalement dans une seule langue. Il serait possible de renforcer la langue non dominante de ces enfants en encourageant les parents à créer des binômes de lecture partagée en dehors du temps scolaire - des parents anglophones pourraient ainsi proposer des lectures partagées en anglais pour les enfants ayant plus de difficultés en anglais, alors que les parents francophones pourraient proposer des lectures en français pour les enfants qui peinent en français. Une telle coopération permettrait de favoriser l'enrichissement progressif de la langue non dominante et l'intérêt des parents pour cette deuxième langue pourrait avoir un effet positif sur l'intérêt de l'enfant lui-même envers cette langue.

\section{Conclusion}

Nous avons observé les effets de l'exposition aux langues sur différentes variables linguistiques en production orale d'enfants bilingues français/anglais. Les résultats révèlent que le vocabulaire réceptif et certaines mesures de productivité narrative semblent être sensibles au degré d'exposition de chaque langue. De plus, nos conclusions renforcent l'idée du rôle essentiel de la lecture sur la performance linguistique, et ce d'autant plus pour les enfants de CP.

Les données présentées dans cet article représentent la première vague de données recueillies. En effet, une deuxième vague de recueil (12 enfants de $\mathrm{CP} ; 18$ enfants de CM2) a été effectuée et est actuellement en cours d'analyse. Il est évident qu'un échantillon plus important permettra d'obtenir des résultats plus robustes. Comme indiqué plus haut, les données présentées proviennent d'un projet longitudinal prévue pour une durée de cinq ans (Cohen, 2015) qui vise à explorer le processus dynamique et complexe de l'acquisition bilingue, en examinant les facteurs qui favorisent son développement et son maintien. Au fur et à mesure de l'avancement de l'étude, nous explorerons l'impact de l'exposition changeante à chaque langue pendant l'enfance et l'adolescence sur l'acquisition de diverses propriétés linguistiques et nous examinerons l'évolution de la relation entre les facteurs qui influencent le développement bilingue et la compétence linguistique lorsque les enfants grandissent.

Nous tenons à remercier tout particulièrement : les enfants et les familles d'avoir accepté de participer à cette étude ; les équipes pédagogiques pour leur intérêt et leur aide tout au long de la collecte des données; le LabEx Aslan (ANR-10-LABX-0081) de l'Université de Lyon dans le cadre du programme «Investissements d'Avenir » (ANR-11-IDEX-0007) de l'État Français géré par l'Agence 
Nationale de la Recherche (ANR), le Laboratoire de l'éducation (LLE, UMS 3773) ainsi que l'Institut des Sciences de L'Homme pour leur soutien financier. Pour finir, nous remercions Eva Soroli pour son minutieux et précieux travail de transcription et codage.

\section{Références bibliographiques}

Aksu-Koç, A. et Küntay, A. (2001). Reformulating causal relations while retelling narratives : evidence from Turkish, Colloque Acquisition et Construction du Sens dans une Perspective Interlangue Paris.

Altman, C., Armon-Lotem, S., Fichman, S. et Walters, J. (2016). Macrostructure, microstructure, and mental state terms in the narratives of English-Hebrew bilingual preschool children with and without specific language impairment. Applied Psycholinguistics, 37, 165-193

Aram, D., Fine, Y. et Ziv, M. (2013). Enhancing parent-child shared book reading interactions: Promoting references to the book's plot and socio-cognitive themes. Early Childhood Research Quarterly, 28, 111-122.

Baker, C. (2006) Foundations of bilingual education and bilingualism ( $4^{\mathrm{e}}$ éd.). Clevedon : Multilingual Matters.

Bedore, L.M., Peña, E.D., Gillam, R.B. et Ho, T-H. (2010). Language Sample Measures and Language Ability in Spanish English Bilingual Kindergarteners. Journal of Communication Disorders. 43(6), 498-510.

Berman, R. et Slobin, D. (1994). Relating events in narrative : a crosslinguistic developmental study. Hillsdale : Erlbaum.

Bianco, M. (2015). Du langage oral à compréhension de l'écrit. Grenoble : PUG.

Bliss, L. S., McCabe, A. et Miranda, E. A. (1998). Narrative assessment profile: Discourse analysis for school-age children. Journal of Communication Disorders, 31, 347-363.

Bohman, T. M., Bedore, L. M., Peña, E. D., Mendez-Perez, A. et Gillam, R. B. (2010). What you hear and what you say: Language performance in Spanish-English bilinguals. International Journal of Bilingual Education and Bilingualism, 13, 325344.

Candéa, M. (2000). Contribution à l'étude des pauses silencieuses et des phénomènes dits " d'hésitations " en français oral spontané (Thèse de doctorat). Université Paris III, Sorbonne Nouvelle.

Catts, H., Adlof, S. M. et Weismer, S. E. (2006). Language deficits in poor comprehenders: a case for the simple view of reading. Journal of Speech, Language and Hearing Research, 49, 278-293.

Cohen, C. (2015). INEXDEB. Lyon, France: Laboratoire Interactions, Corpus, Apprentissages et Représentations.

Cohen, C. (2016). Relating input factors and dual language proficiency in French-English bilingual children. International Journal of Bilingual Education and Bilingualism, 19(3), 296-313.

Cummins, J. (1978). Bilingualism and the development of metalinguistic awareness. Journal of Cross-Cultural Psychology, 9, 131-149.

De Houwer, A. (2011). Language input environments and language development in bilingual acquisition. Applied Linguistics Review, 2, 221-240.

De Houwer, A. (2015). Harmonious bilingual development: Young families' well-being in language contact situations. International Journal of Bilingualism, 19(2), 169-184.

DeTemple, J. M. (2001). Parents and children reading books together. Dans D.K. Dickinson et P.O. Tabors (dir.), Beginning literacy with language (p. 33-51). Baltimore : Brookes Publishing.

Dunn, L.M., Dunn, L.M., Whetton, C. et Burley, J. (1987). The British Picture Vocabulary Scale ( ${ }^{\mathrm{e}}$ éd.). Windsor : NFER-Nelson. 
Dunn, L.M., Thérault-Whalen, C.M. et Dunn, L.M. (1993). Echelle de vocabulaire en images Peabody. Toronto: Psycan.

Fayol, M. (1984). La distanciation dans le langage, Enfance, 1, 5-19.

Fayol, M. (1997). Des idées au texte : psychologie cognitive de la production verbale orale et écrite. Paris: Presse Universitaire de France.

Fayol, M. (2000). Comprendre et produire des textes écrits : l'exemple du récit. Dans M. Kail et M. Fayol (dir.), L'acquisition du langage, le langage en developpement audelà de trois ans (p. 183-214). Paris : Presses Universitaires de France.

Fishman, J. (dir.) (2001). Can threatened languages be saved: Reversing language shift, revisited. Clevedon, UK : Multilingual Matters.

Gagarina, N., Klop, D., Tsimpli, I. M. et Walters, J. (2016). Narrative abilities in bilingual children. Applied Psycholinguistics, 37, 11-17.

Gardner-Neblett, N. et Iruka, I. U. (2015). Oral narrative skills: Explaining the languageemergent literacy link by race/ethnicity and SES. Developmental Psychology, 51(7), 889-904.

Gathercole, V.C.M. (2007). Miami and North Wales, so far and yet so near: a constructivist account of morphosyntactic development in bilingual children. International Journal of Bilingual Education and Bilingualism, 10(3), 224-247.

Goldberg, H., Paradis, J. et Crago, M. (2008). Lexical acquisition over time in minority first language children learning English as a second language. Applied Psycholinguistics, 29, 41-65.

Herdina, P. et Jessner, U. (2002). A dynamic model of multilingualism. Clevedon : Multilingual Matters.

Hickmann, M. (2003). Children's discourse: person, space and time across languages. New York: Cambridge University Press.

Iluz-Cohen, P. et Walters, J. (2012). Telling Stories in two Languages: Narratives of Bilingual Preschool Children with Typical and Impaired Language. Bilingualism : Language and Cognition, 15, 58-74.

Jia, G. et Aaronson, D. (2003). A longitudinal study of Chinese children and adolescents learning English in the United States. Applied Psycholinguistics, 24, 131-161.

Jisa, H. (2000). Increasing cohesion in narratives: a developmental study of maintaining and reintroducing subjects in French. Linguistics, 38, 591-620.

Jisa, H. et Mazur, A. (2006). L'expression de la causalité : une étude développementale, Paper presented at the Journée d'étude : Des savoirs savants aux savoirs enseignés. Université Paris X, Nanterre, 33-60.

Labov, W. (1993). Le parler ordinaire, la langue dans les ghettos noirs des Etats-Unis. Paris : Les éditions de minuit.

Lonigan CJ, Schatschneider C et Westberg L. (2008). Developing Early Literacy: Report of the National Early Literacy Panel. Washington, DC : National Institute for Literacy; Identification of children's skills and abilities linked to later outcomes in reading, writing, and spelling (p. 55-106).

MacWhinney, B. (2000). The CHILDES Project: Tools for Analyzing Talk (3 $3^{\mathrm{e}}$ éd.). Mahwah, NJ : Lawrence Erlbaum Associates.

Mayer, M. (1969). Frog, where are you? New York : Dial Press.

Mol, S. E., Bus, A. G. et de Jong, M. T. (2009). Interactive book reading in early education: A tool to stimulate print knowledge as well as oral language. Review of Educational Research, 79, 979-1008.

Mol, S. E. et Bus, A. G. (2011). To read or not to read : A meta-analysis of print exposure from infancy to early adulthood. Psychological Bulletin, 137(2), 267-296.

Montanari, S. (2004). The development of narrative competence in the L1 and L2 of Spanish-English bilingual children. International Journal of Bilingualism, 4, 449497. 
NICHD Early Child Care Research Network. (2005). Pathways to reading: The role of oral language in the transition to reading. Developmental Psychology, 41(2), 428-442.

Oller, D.K. et Eilers, R.E. (dir.) (2002). Language and literacy in bilingual children. Clevedon: Multilingual Matters.

Osborn, M. (2009). Etre élève en Angleterre et en France. Revue Internationale d'Education de Sèvres, 50, 87-98.

Paradis, J. (2011). Individual differences in child English second language acquisition: Comparing child-internal and child-external factors. Linguistic Approaches to Bilingualism, 1, 213-237.

Paradis, J., Emmerzael, K. et Duncan, T.S. (2010). Assessment of English language learners: Using parent report on first language development. Journal of Communication Disorders, 43, 474-497.

Pearson, B. Z. (2002). Narrative competence in bilingual school children in Miami. Dans D. K. Oller et R. E. Eilers (dir.), Language and literacy in bilingual children. Clevedon : Multilingual Matters.

Pearson, B. Z. (2007). Social factors in childhood bilingualism in the United States. Applied Psycholinguistics, 28(3), 399-410.

Pearson, B.Z. (2008). Raising a bilingual child. New York : Living Language.

Pearson, B.Z. et Amaral, L. (2014). Interactions between input factors in bilingual language acquisition : Considerations for minority language maintenance. Dans T. Grüter et $\mathrm{J}$. Paradis (dir.), Input and experience in bilingual development. Amsterdam : John Benjamins.

Pearson, B.Z., Fernández, S.C., Lewedeg, V. et Oller, D.K. (1997). The relation of input factors to lexical learning by infant bilinguals. Applied Psycholinguistics, 18, 41-58.

Pollard-Durodola, S.D., Gonzalez, J.E., Simmons, D.C. et Simmons, L. (2015). Accelerating language skills and content knowledge through shared book reading. Baltimore, MD : Paul H. Brookes.

Ravid, D. (2000). NP complexity in the development of text writing, Developing Literacy Across Genres, Modalities and Languages, 3, 163-170

Rodina, Y. (2017). Narrative abilities of preschool bilingual Norwegian-Russian children. International Journal of Bilingualism, 21(5), 617-635.

Rojas, R. et Iglesias, A. (2013). The Language Growth of Spanish-Speaking English Language Learners. Child Development, 84(2), 630-646.

Scheidnes, M. et Tuller, L. (2016). Assessing successive bilinguals in two languages: a longitudinal look at English-speaking children in France. Journal of Communication Disorders, 64, 45-61.

Schneider, P., Hayward, D. et Dubé, R.V. (2006). Storytelling from pictures using the Edmonton Narrative Norms Instrument. Journal of Speech Language Pathology and Audiology, 30, 224- 238.

Sénéchal, M. (2006). Testing the home literacy model: Mother involvement in kindergarten is differentially related to grade 4 reading comprehension, fluency, spelling, and reading for pleasure. Scientific Studies of Reading, 10, 59-87.

Sénéchal, M. et Kearnan, K. (2007). The role of morphology to reading and spelling. Dans R. Kail (dir.), Advances in child development and behavior (vol. 35, p. 297-325). San Diego, CA : Elsevier Academic Press.

Sénéchal, M., Pagan, S., Lever, R. et Ouellette, G.P. (2008). Relations Among the Frequency of Shared Reading and 4-Year-Old Children's Vocabulary, Morphological and Syntax Comprehension, and Narrative Skills. Early Education and Development, 19(1), 27-44.

Shanahan, T. et Lonigan, C.J. (2013). Early Childhood Literacy: The National Early Literacy Panel and Beyond. Paul H. Brookes Publishing Company : Baltimore. 
Simon-Cereijido, G. et Gutiérrez-Clellen, V. F. (2009). A cross- linguistic and bilingual evaluation of the interdependence between lexical and grammatical domains. Applied Psycholinguistics, 30, 315-337.

Thordardottir, E. (2011). The relationship between bilingual exposure and vocabulary development. International Journal of Bilingualism, 15, 426-445.

Thordardottir, E., Rothenberg, A., Rivard M.-E. et Naves, R. (2006). Bilingual Assessment: Can Over-all Proficiency Be Estimated from Separate Assessment of Two Languages? Journal of Multilingual Communication Disorders, 4, 1-21.

Uccelli, P. et Paéz, M.M. (2007). Narrative and Vocabulary Development of Bilingual Children From Kindergarten to First Grade: Developmental Changes and Associations among English and Spanish Skills. Language Speech and Hearing Services in Schools $38,225-236$.

Unsworth, S. (2015). Quantity and quality of language input in bilingual language development.' Dans E. Nicoladis, E. et S. Montanari (dir.), Lifespan perspectives on bilingualism (p. 136-196). Washington, D.C. : Mouton de Gruyter.

van Kleek, A. et Stahl, S.A. (2003). Preface. Dans A. van Kleeck, S.A. Stahl et E. Bauer (dir.), On reading to children : Parents and teachers (p. vii-xiii). New York: Lawrence Erlbaum Associates.

Weizman Z.O. et Snow C.E. (2001). Lexical input as related to children's vocabulary acquisition: Effects of sophisticated exposure and support for meaning. Developmental Psychology, 37, 265-279.

Wigglesworth, G. (1991). Reference tracking strategies of young children in extended narrative discourse, Paper presented at the Working papers in Linguistics, La Trobe University.

Yuan, F. et R. Ellis (2003). The effects of pre-task planning and on-line planning on fluency, complexity and accuracy in L2 oral production. Applied Linguistics, 24, 1-27.

\section{Annexe 1}

Tableau 13. Statistiques descriptives mesures relatives à l'exposition : CP anglais (CP A), $\mathrm{CP}$ français (CP F), CM2 anglais (CM2 A), CM2 français (CM2 F)

\begin{tabular}{|c|c|c|c|c|}
\hline Groupe & & $\begin{array}{l}\text { Exposition courante } \\
\text { (\% du temps) }\end{array}$ & $\begin{array}{l}\text { Exposition cumulative } \\
\text { (nombre d'années) }\end{array}$ & $\begin{array}{c}\text { Lecture** } \\
\text { (nombre de fois / an) }\end{array}$ \\
\hline \multirow[t]{3}{*}{ CP A } & $M$ & 57.49 & 3.94 & 306.67 \\
\hline & $E ́ T$ & 14.65 & 1.18 & 93.81 \\
\hline & Intervalle & $31.69-73.12$ & $2.1-5.8$ & $80-350$ \\
\hline \multirow[t]{3}{*}{ CP F } & $M$ & 42.39 & 2.06 & 156.44 \\
\hline & $\vec{E} T$ & 14.56 & 1.18 & 161 \\
\hline & Intervalle & 26.88 & $.2-3.9$ & $0-350$ \\
\hline \multirow[t]{3}{*}{ CM2 A } & $M$ & 42.54 & 4.97 & 293.2 \\
\hline & $E ́ T$ & 20.21 & 2.26 & 110.28 \\
\hline & Intervalle & $15.39-78.78$ & $2.15-9.4$ & $8-350$ \\
\hline \multirow[t]{3}{*}{ CM2 F } & $M$ & 52.43 & 4.19 & 278 \\
\hline & $E ́ T$ & 20.16 & 2.39 & 140.47 \\
\hline & Intervalle & $21.22-84.61$ & $.6-7$ & $20-350$ \\
\hline
\end{tabular}

** CP lecture partagée ; CM2 lecture en autonomie

Tableau 14. Statistiques descriptives mesures linguistiques : CP anglais (CP A), CP français (CP F), CM2 anglais (CM2 A), CM2 français (CM2 F)

\begin{tabular}{|l|l|c|c|c|c|c|c|c|c|}
\hline Groupe & $\begin{array}{c}\text { Vocab } \\
\text { réceptif* }\end{array}$ & NMD & NVD & Erreurs & $\begin{array}{c}\text { Phrases } \\
\text { complexes }\end{array}$ & MDTF & $\begin{array}{c}\text { Mots / } \\
\text { minute }\end{array}$ & $\begin{array}{c}\text { Pauses } \\
\text { vides }\end{array}$ \\
\hline CP A & $M$ & 106.1 & 2.89 & .72 & .02 & .19 & .05 & 91.62 & .44 \\
\hline & $\hat{E} T$ & 11.45 & .6 & .13 & .01 & .14 & .04 & 23.26 & .06 \\
\hline
\end{tabular}


SHS Web of Conferences 46, 10004 (2018)

https://doi.org/10.1051/shsconf/20184610004

Congrès Mondial de Linguistique Française - CMLF 2018

\begin{tabular}{|l|l|c|c|c|c|c|c|c|c|}
\hline & Inter & $89-124$ & $1.88-3.83$ & $.55-.93$ & $0-.05$ & $0-.5$ & $.01-.17$ & $45-126$ & $.35-.52$ \\
\hline CP F & $M$ & 108.89 & 2.84 & .73 & .04 & .19 & .09 & 79.36 & .47 \\
\hline & ÉT & 19.08 & 1.1 & .25 & .03 & .18 & .05 & 22.23 & .11 \\
\hline & Inter & $75-128$ & $1.67-5.43$ & $.4-1.29$ & $.01-.09$ & $0-.57$ & $.03-.18$ & $45-114$ & $.32-.65$ \\
\hline CM2 A & $M$ & 110 & 3.24 & .94 & .006 & .18 & .04 & 114.96 & .39 \\
\hline & ÉT & 13.97 & .69 & .22 & .008 & .15 & .02 & 20.14 & .07 \\
\hline & Inter & $77-135$ & $2.33-4.73$ & $.68-1.53$ & $0-.03$ & $0-.6$ & $.01-.07$ & $84-148$ & $.24-.52$ \\
\hline CM2 F & $M$ & 105.67 & 2.95 & .75 & .02 & .11 & .06 & 106.33 & .40 \\
\hline & ÉT & 14.99 & .71 & .71 & .02 & .08 & .03 & 19.53 & .07 \\
\hline & Inter & $75-132$ & $2.06-5.03$ & $.48-1.36$ & $0-.06$ & $.04-.31$ & $.03-.12$ & $74-144$ & $.27-.51$ \\
\hline
\end{tabular}

* anglais=BPVS ; français=EVIP 